intensities of all the observed planes. In this structure the asymmetric unit is a half molecule of approximate diameter $35 \mathrm{~A}$, linked with three others. The molecule observed in solution may be formed of two of these. The optical properties of the wet crystal point to the approximate parallelism of the hæmatin groups which lie approximately parallel to the (100) planes. As yet their position cannot be inferred, but owing to the two-fold axis of symmetry which the double molecule possesses, only two different kinds of hæmatin globin arrangements are possible. In the meantime, it is possible to draw some useful conclusions from the character of the photographs. In both cases the wet crystals showed perfectly definite reflections at spacings as low as $2 \mathrm{~A}$. This proves the complete internal regularity of the protein molecules down to atomic dimensions. The dry crystals, on the other hand, show in the case of hæmoglobin no reflections of spacing less than $20 \mathrm{~A}$, and for chymotrypsin, except for one or two weak reflections at $5 \mathrm{~A}$., none below $8 \mathrm{~A}$. Dr. Crowfoot had already noticed this phenomenon for insulin and more recently for lactoglobulin. The explanation is probably somewhat as follows. The intensity of a reflection from a protein crystal may be considered a function of three factors : the structure factor due to the position of the molecules, that due to the positions of the atoms inside the molecule, and a third factor depending on the regularity of arrangement. It is apparent that there is a general enhancement of spots in the regions of $9 \mathrm{~A}$. and $4.5 \mathrm{~A}$. corresponding to the two main reflections maxima of denatured proteins. In the dry protein, however, the irregularity is such that only the first of these is retained, the spots corresponding to the outer rim being generally too weak to register. If this analysis is correct, it follows that the change involved on denaturation does not require any considerable movement of atoms or amino-acid residues, but only relatively minor rearrangement together with almost complete loss of regularity, the dry protein representing an intermediate stage.

As can be seen from Fig. 2 the dried crystals of chymotrypsin show not only alterations of spacing but also of relative intensities of reflection. If we assume that drying takes place by the removial of water from between protein molecules, studies of these changes provide an opportunity of separating the effects of inter-and intra-molecular scattering. This may make possible the direct Fourier analysis of the molecular structure once complete sets of reflections are available in different states of hydration.

Already sufficient protein crystals have been examined by $\mathrm{X}$-rays to be able to point to significant regularities. The table of lattice dimensions referred to three axes at right angles shows that the proteins fall into two groups: pseudo-hexagonal and pseudocubic, in both cases with a side of 60-75 A. This is of the order of twice the diameter of a protein molecule.

The work already done shows the wealth of new data that can be obtained by $\mathrm{X}$-ray methods from reasonably well-crystallized proteins. The full advantage of the possibilities can, however, only be realized by work on a far larger number of protein types under different physical conditions, together with a more detailed application of crystal analysis.

\footnotetext{
1 J. Biol. Chem., 104, 359 (1934).

${ }^{2}$ Proc. Roy. Soc., B, 120, 422 (1936).

sedersen, K. O., Biochem. J., 30, 961 (1936).

+ Proc. Roy. Soc., A., 164, 580 (1938).

'J. Chem. Soc., 849 (1935).

Chem. Rev., 20, 81 (1937).

'Bernal, J. D., and Crowfoot, D., NATURE, 133, 794'(1934).

"Reichert and Brown, "The Crystallography of Hemoglobin". Carnegie Inst. of Washington (1909).

'Chick and Martin, Biochem. J., 7, 92 (1913).

10 Haurowitz, F., Z. Physiol. Chem., 136, 147.
}

\title{
Asymmetric-Sideband Broadcasting
}

A T a meeting of the Wireless Section of the Institution of Electrical Engineers on March 2, Mr. P. P. Eckersley read a paper entitled" "A Quantitative Study of Asymmetric-Sideband Broadcasting". This paper directed attention to the fact that the progress of radio broadcasting is being hampered by the lack of a sufficient number of channels in which to contain the increasing number of transmissions. In the present state of the art, few, except local, stations can be received without interference from other broadcasting stations on adjacent frequency channels, unless the upper modulation frequencies of the wanted programme are severely attenuated. This inter-station interference can be reduced if all or part of one sideband of the transmitted spectrum is cut away; and it is the object of the asymmetricsideband system of transmission to cut away part of one sideband without loss of the higher modulation frequencies and without introducing audible harmonic distortion.

Mr. Eckersley then gave a quantitative analysis of the phase and amplitudes of the sideband components and the effect of these factors in producing distortion. Curves have been derived, which show the required attenuation-constant of filters which cut away part of one sideband but produce no more than a constant, small distortion. A description was also given of practical tests in which the quality of reproduction obtainable from an asymmetric transmitter was compared with that given by orthodox modulation. It is claimed that while a highly trained ear may occasionally be able to detect some slight differences between the two types of reproduction when they are directly compared, no difference is detectable with modern broadcast receiving apparatus.

The last part of the paper showed that the asymmetric system has three possible applications to broadcasting as it is practised to-day. First, it could be used to allow carrier frequency differences of 11 or $12 \mathrm{kc} . / \mathrm{sec}$. without introducing any sideband overlap interference. Secondly, it could be applied to existing conditions in which the carrier frequencies differ by about $9 \mathrm{kc} . / \mathrm{sec}$., and it would then reduce inter-station interference to about one tenth of its present value. Thirdly, if the existing interferences are considered to be tolerable, then the differences between the carrier-frequencies of stations can be reduced to $6 \mathrm{kc} . / \mathrm{sec}$., without increasing the interference; this implies that one and a half times the number of stations working in Europe to-day on exclusive wave-length channels could be accommodated in the existing wave-band. 